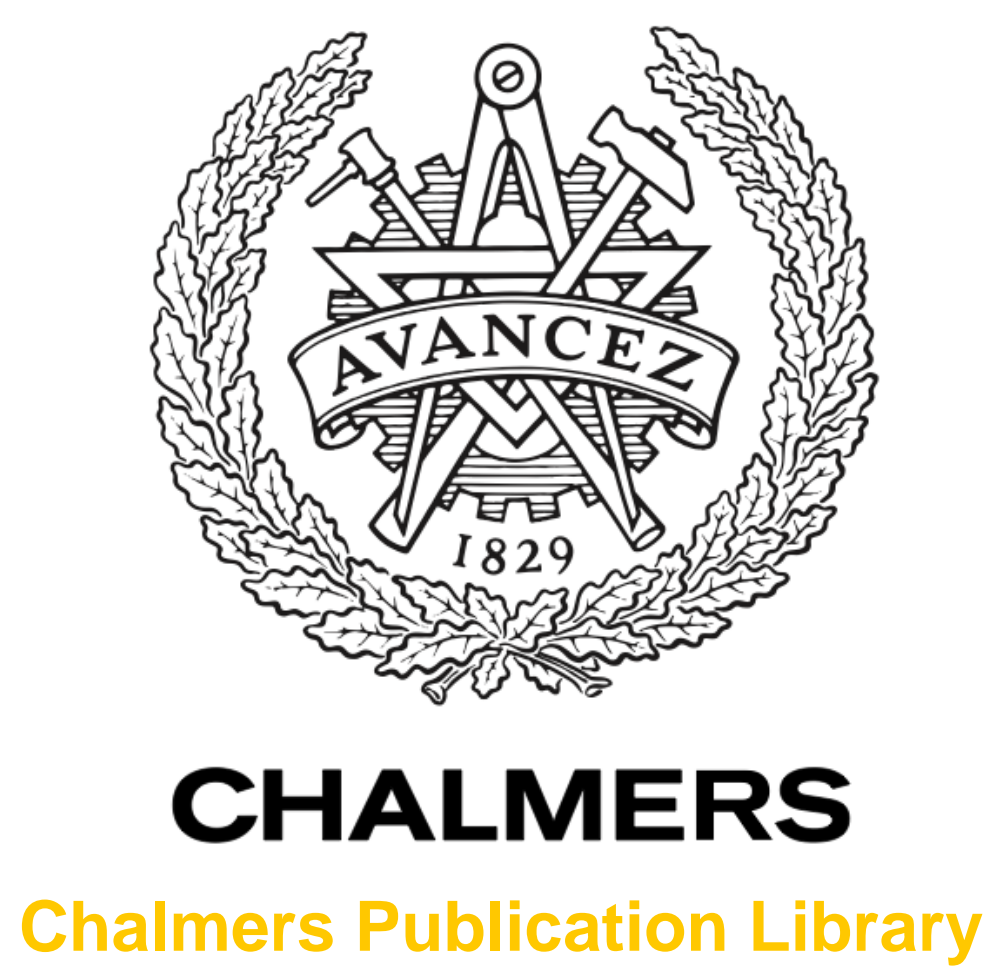

\title{
AWARDS: Advanced microwave radiometers for deep space stations
}

This document has been downloaded from Chalmers Publication Library (CPL). It is the author's version of a work that was accepted for publication in:

Space communications (ISSN: 0924-8625)

Citation for the published paper:

Tortora, P. ; Crewell, S. ; Elgered, G. (2013) "AWARDS: Advanced microwave radiometers for deep space stations". Space communications, vol. 22(2-4), pp. 159-170.

http://dx.doi.org/10.3233/SC-130011

Downloaded from: http://publications.lib.chalmers.se/publication/187946

Notice: Changes introduced as a result of publishing processes such as copy-editing and formatting may not be reflected in this document. For a definitive version of this work, please refer to the published source. Please note that access to the published version might require a subscription.

Chalmers Publication Library (CPL) offers the possibility of retrieving research publications produced at Chalmers University of Technology. It covers all types of publications: articles, dissertations, licentiate theses, masters theses, conference papers, reports etc. Since 2006 it is the official tool for Chalmers official publication statistics. To ensure that Chalmers research results are disseminated as widely as possible, an Open Access Policy has been adopted.

The CPL service is administrated and maintained by Chalmers Library. 


\title{
AWARDS: ADVANCED MICROWAVE RADIOMETERS IN DEEP SPACE STATIONS
}

\author{
ESA/ESTEC, NOORDWIJK, THE NETHERLANDS \\ 30 NOVEMBER - 2 DECEMBER 2011
}

\author{
Paolo Tortora $^{(1)}$, Susanne Crewell ${ }^{(2)}$, Gunnar Elgered ${ }^{(3)}$, Alberto Graziani ${ }^{(1)}$, Per Jarlemark ${ }^{(4)}$, Ulrich Loehnert $^{(2)}$, Antonio \\ Martellucci $^{(5)}$, Mattia Mercolino ${ }^{(6)}$, Thomas Rose $^{(7)}$ and Jan Schween ${ }^{(2)}$ \\ ${ }^{(1)}$ Department of Industrial Engineering, University of Bologna, Via Fontanelle, 40, 47121, Forli, Italy \\ Email: paolo.tortora@unibo.it,alberto.graziani@unibo.it \\ ${ }^{(2)}$ Institute of Geophysics and Meteorology, University of Cologne, Zülpicher Str. 49a, 50674, Cologne, Germany \\ Email: crewell@meteo.uni-koeln.de,loehnert@meteo.uni-koeln.de,jschween@uni-koeln.de \\ (3) Department of Earth and Space Sciences, Chalmers University of Technology, S-439 92 Onsala, Sweden \\ Email: gunnar.elgered@chalmers.se \\ ${ }^{(4)}$ SP Technical Research Institute of Sweden, Box 857, SE-501 15 Borås, Sweden Email: per.jarlemark@sp.se \\ ${ }^{(5)}$ European Space Agency, ESTEC, TEC-EEP, Keplerlaan. 1, NL-2200 AG Noordwijk, The Netherlands \\ Email: Antonio.Martellucci@esa.int \\ ${ }^{\left({ }^{6}\right)}$ European Space Agency, ESOC,Darmstadt, Germany,Email: mattia.mercolino@esa.int \\ (7) Radiometer Physics GmbH, Meckenheim, Birkenmaarstrasse 10, 53340, Meckenheim, Germany \\ Email:rose@radiometer-physics.de
}

\begin{abstract}
The objective of this study, named AWARDS (Advanced microwave Radiometers in Deep space Stations), is the preliminary design of a transmission Media Calibration System (MCS) to be located at an ESA Deep Space Antenna (DSA) site. The crucial aspect is the capability to accurately retrieve the tropospheric path delay along the line-of-sight of the deep space probe in order to allow precise tropospheric calibration of deep space observables (range and rangerate) with particular reference to the BepiColombo spacecraft and its primary DSA at Cebreros (ES).

The study focuses on two main aspects which lead to the preliminary design of the Mercury Orbiter Radioscience Experiment (MORE) MCS: the characterization of current microwave radiometers (MWRs) available at ESA/ESTEC and the atmospheric fluctuation effects on the MCS error budget, in terms of the Allan standard deviation (ASD).

In the course of the study, further critical aspects have been identified (effects of Sun contamination, effects of ground noise emission), and mitigation strategies have been proposed.

The final outcome is a preliminary design of the MWR (and the entire MCS) to be deployed at the ESA/ESTRACK (ESA Tracking station network) sites and being compliant with MORE requirements.
\end{abstract}

\section{INTRODUCTION}

We present the main activities and results obtained in the ESA contract: Advanced microWAve Radiometers in Deep space Stations (AWARDS) [1].

Precise microwave tracking systems are a key tool for planetary exploration: the use of Ka-band and multifrequency systems, in particular, was proven to increase the level of accuracy for a variety of scientific objectives ranging from gravity science to relativity studies, from atmospheric radio occultations to bistatic radar experiments [2] [3].

The NASA Cassini mission was the first mission endowed with a multifrequency link designed to cancel out the effects of charged particles in the solar corona interplanetary plasma and the Earth ionosphere. This has allowed to carry out two of the most precise experimental test of general relativity performed so far: the Cassini Gravitational Waves Experiment (GWE) and the Cassini Solar Conjunction Experiment (SCE) ([4], [5]).

Following these successes, the next ESA mission to Mercury, BepiColombo, will carry onboard the Mercury Orbiter Radioscience Experiment - MORE [6]. This experiment makes use of a digital transponder at Kaband, where a ranging channel capable of measurements as accurate as $20 \mathrm{~cm}$, with an integration time of a few seconds, was added to the Doppler channel expected to provide two-way range-rate measurements accurate to 3 $\mu \mathrm{m} / \mathrm{s}$, at $1000 \mathrm{~s}$ observation time. MORE has precise requirements in terms of the error contribution to the end-to-end error budget for the ground segment and the residual uncalibrated media noises. In particular, the antenna mechanical noise and the residual tropospheric noise are among the major noise sources for deep space tracking [7], once the solar and interplanetary plasma is removed thanks to the multifrequency link configuration.

Current MORE error budgets require the residual tropospheric noise to be less than $2 \mathrm{~cm}$ on medium-long time scales for ranging observables, and $8 \times 10^{-15} \mathrm{~s} / \mathrm{s}$ at $1000 \mathrm{~s}$ (expressed in terms of Allan Standard Deviation 
- ASD) [8] for range rate observables. In this study, MORE requirements have been revised and renamed as AWARDS requirements. As far as the AWARDS requirements are concerned, the following values have been considered: $3 \times 10^{-14} \mathrm{~s} / \mathrm{s}$ at $20 \mathrm{~s}, 3 \times 10^{-15} \mathrm{~s} / \mathrm{s}$ at 1000 $\mathrm{s}$ and $3 \times 10^{-15} \mathrm{~s} / \mathrm{s}$ at $10000 \mathrm{~s}$.

As shown in Figure 1, compared to the MORE requirements the AWARDS ones are more severe at $1000 \mathrm{~s}$ and $10000 \mathrm{~s}$. This revision was decided to evaluate the technological limits and to reduce as much as possible the impact of the troposphere component on the entire MORE error budget. A smaller impact of the troposphere component might also mitigate some limitations in other components (e.g. the spacecraft segment).

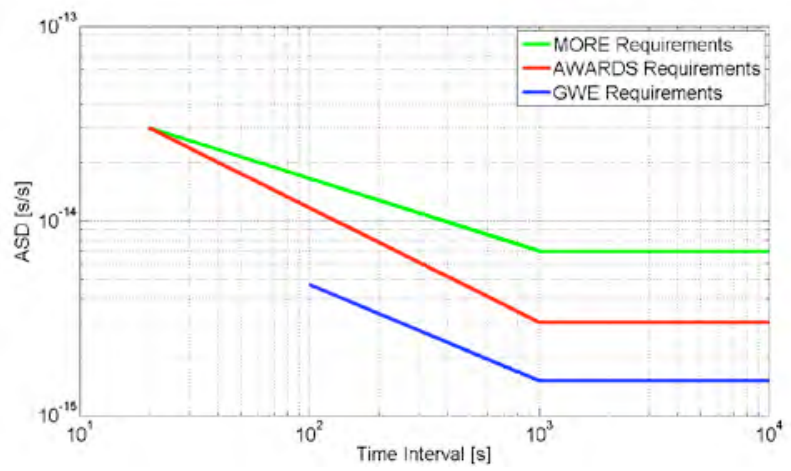

Figure 1: Upper-bounds of the ASD requirements for MORE (red), AWARDS (green) and Cassini's GWE (blue)

The very stringent AWARDS requirements can be met only by using a dedicated instrument, the MCS incorporating an ultra-stable MWR, capable to estimate the Slant Wet Delay (SWD) [9] along the DSA-probe line of sight.

A crucial aspect of the AWARDS study is the evaluation of current MWR performance in terms of ASD. For this purpose two ESA MWRs were compared: the RPG-ATPROP (Atmospheric Profiler) [10] and the RESCOM, both based on Dicke switch technology [11] and channels in the same main water vapor and liquid bands $(23.8 \mathrm{GHz}$ and $31.4 \mathrm{GHz})$.

Together with a preliminary design of the MCS, the evaluation of the performance of the MWR were the core of the AWARDS study.

\section{TECHNIQUES FOR THE STABILITY CHARACTERIZATION OF MICROWAVE RADIOMETERS}

The radio science experiment requirements are defined in terms of two-way tracking ASD at different observation times. The ASD is defined for the statistical analysis of the stability of atomic time oscillators. Further developed studies demonstrated that the theory can be applied to other time series, and it is particularly useful in the statistical characterization of the stability of a Doppler link [12]. Moreover, it is also widely used in the context of other noise processes, such as instrumental radiometric noise [13], [14].

In the following paragraphs both a description of the computation process of the ASD and three methods used in the study to characterize the MWR internal noise are presented.

A definition of ASD, as given in [15], is particularly useful for the estimation of the stability of the measured troposphere path delay:

$$
\sigma_{y}(\tau)=\left(\left\langle\frac{(x(t+2 \tau)-2 x(t+\tau)+x(t))^{2}}{2 \tau^{2}}\right\rangle\right)^{1 / 2}[\mathrm{~s} / \mathrm{s}](1)
$$

Where $\mathrm{x}$ is the path delay in seconds at time $t, \tau$ is the Doppler observation time. Equation (1) defines a statistical quantity which is proportional to the secondorder variability of the delay over $\tau$. The path delay $x$ is obtained from the PD measurements simply by dividing by the speed of light.

A crucial characteristic of Eq. (1) is that the presence of a bias and a drift in the data does not affect the result in terms of ASD. Moreover, for short timescales $\tau$ (up to $1000 \mathrm{~s}$ ), the model of the residual path delay due to the MWR internal noise can be modeled as a combination of a linear drift, a bias and a White Gaussian Noise (WGN) of standard deviation $\sigma_{n}$ [15]. With this approximation the ASD can be simplified to:

$$
\sigma_{y}(\tau)=\frac{\sqrt{3} \cdot \sigma_{n}}{\tau}[\mathrm{s} / \mathrm{s}]
$$

\subsection{Theoretical Internal Noise Computation}

Starting from Eq. (2) the standard deviation $\sigma_{n}$ can be defined as the radiometric resolution of the water vapor channel. Considering the generic equation of the radiometric resolution of a noise injection Dicke switch MWR like ATPROP [16]:

$$
\sigma_{\text {dicke }}(\Delta t)=2 \frac{T_{R E F}+T_{R}}{\sqrt{B \Delta t}}[\mathrm{~K}]
$$

Where: $\Delta t$ is the MWR integration time, $T_{R E F}$ is the temperature of the reference load, $T_{R}$ is the receiver noise temperature and $B$ is the bandwidth.

$$
\sigma_{n}(\Delta t)=\sigma_{\text {dicke }}(\Delta t) \cdot v_{n}[\mathrm{~s}]
$$

Where $n$ represents the water vapor channel and $v$ is the radiometric sensitivity, which is in the order of $2 \times 10^{-11}$ $\mathrm{s} / \mathrm{K}$ for the $23.8 \mathrm{GHz}$ channel.

By replacing Eq. (4) in Eq. (2) it is possible to estimate the theoretical internal noise of the instrument in terms of ASD starting from the design MWR parameters.

\subsection{Difference procedure}

This technique named "Difference Procedure" has been defined to characterize the internal noise of a MWR based on the measurements of another radiometer. The two MWRs have to be installed close 
to each other and pointing in the same direction. The main idea is that apart from all possible instrumental differences, the two instruments are observing the same atmosphere.

For each instrument, the estimated $S W D$ can be separated in the sum of two contributions: a $S W D$ due to the atmosphere, $S W D_{a t m}$, and a contribution due to the instrumental internal noise, $S W D^{I}$. Labeling the instruments with the subscript $A$ (the instrument to be characterized) and $B$, the sum of the $W P D$ can be written as:

$$
\left.\begin{array}{l}
S W D_{A}=S W D_{a t m}+S W D_{A}^{I} \\
S W D_{B}=S W D_{a t m}+S W D_{B}^{I}
\end{array}\right\}
$$

The procedure is based on the use of the $A S D$ of the difference of the two instruments' $S W D$. This means that, according to the previous hypothesis, the tropospheric contribution cancels out while the sum (in a quadratic sense) of the instrumental internal noise remains. The $A S D$ of the difference of the two signals is defined as:

$$
\triangle A S D_{A B}(\tau)=A S D\left(S W D_{B}(t)\right)-A S D\left(S W D_{A}(t)\right)
$$

Replacing the terms of equation (5) in equation (4), expanding and simplifying, it results:

$$
\triangle A S D_{A B}(\tau)=\sqrt{A S D_{B}^{I}(\tau)^{2}+A S D_{A}^{I}(\tau)^{2}}
$$

where $A S D_{A}{ }^{I}$ and $A S D_{B}{ }^{I}$ are the $A S D$ due to internal noise of instrument $A$ and $B$, respectively. Since the two instruments are different it is assumed that instrument $B$ is $N$ times more noisy than is instrument $A$ (which is the instrument to be characterized):

$$
A S D_{B}^{I}(\tau)=N \cdot A S D_{A}^{I}(\tau)
$$

The term $N$ is independent of $\tau$. It is also verified that, if the internal noise is white Gaussian, its $A S D$ follows a $\tau^{-1}$ power law. An analysis of the instrument pure internal noise (not reported here) shows that the $A S D$ follows a $\tau^{-1}$ power law. Because of this behavior, the ratio $N$ can be considered constant.

Replacing Equation (7) in Equation (6) it results:

$$
\triangle A S D_{A B}(\tau)=A S D_{A}^{I}(\tau) \cdot \sqrt{N^{2}+1}
$$

A further assumption is that the total $A S D$ derived from the measured signal time series can be assumed to represent only the instrument internal noise, if small observation times and clear sky conditions are considered:

$$
A S D\left(\tau_{0}\right) \cong A S D^{I}\left(\tau_{0}\right)
$$

Thus the ratio $N$ can be derived from the $A S D$ at the smallest available instrument integration time $\tau_{0}$, which coincide with the time series temporal step. Computing the $A S D$ of the signal measured by the two instruments at the same (short) integration time $\tau_{0}$, the ratio $N$ can be computed as:

$$
N=N_{0}=A S D_{B}\left(\tau_{0}\right) / A S D_{A}\left(\tau_{0}\right)
$$

Once the $N_{0}$ term is computed, it can be replaced in equation (8) to compute the $A S D_{A}^{I}(\tau)$ term.

\subsection{White noise procedure}

This procedure is based on the data of a single instrument and the assumption that the internal noise is a pure WGN. If that is the case, then the ASD of a WGN follows a $\tau^{-1}$ power law.

Moreover, following equation (9) the ASD of the data series at the smallest time step is dominated by ASD of the instrumental noise. This assumption is valid if the data have been acquired in clear sky conditions where, at short integration time, the ASD is dominated by the instrumental internal noise. For this reason this procedure has been named "White Noise Procedure" (WNP). Using these two assumptions together, the ASD of the instrumental noise at any observation time $\tau$ is computed as:

$$
A S D(\tau)=A S D\left(\tau_{0}\right) \cdot\left(\frac{\tau}{\tau_{0}}\right)^{-1}
$$

\section{CHARACTERIZATION OF THE ATMOSPHERIC VARIABILITY INFLUENCE}

In this section the effect of the atmospheric variability due to the different beams and positions of the DSA and the MWR is discussed.

The parameter of interest is the slant delay of a signal propagating through the Earth's atmosphere to a receiver on the ground. The variations in the propagation delay in the microwave region are dominated by water vapor. Therefore, the focus will be on the wet refractivity and the SWD. It should, however, be noted that the calibration requirement is to correct at least for $90 \%$ of the slant wet delay. Therefore the variations in the dry refractivity (mainly temperature variations) may become equal in size with the wet delay residual after calibration.

For an accurate calibration of the SWD along the signal path, the DSA and the MWR beams should ideally be identical. A deviation from identical beams could be due to one or a combination of the following reasons: the MWR antenna beam is wider than the DSA beam, the site position of the MWR is offset with respect to the DSA beam, there is a pointing offset of the MWR on the sky, e.g., in order to avoid radiation from the sun or the ground. A different SWD is then obtained from the MWR data which are then to be used to calibrate the SWD in the DSA observations.

We have developed software to quantify the expected sizes of the differential SWD variability between the two beams for different observation geometries. It is based on the statistical model presented in [17]. The model starts with a turbulence theory description of the wet refractivity variations that is integrated to produce the wet delay variability structure 
functions, both in time and in space. In our applications we use standard values for the model parameters, e.g., the strength of the turbulence $C_{n}^{2}$ is fixed to $5.76 \cdot 10^{-14} \mathrm{~m}^{-2 / 3}$, together with an atmospheric thickness of $1 \mathrm{~km}$. We calculate the ASD as well as the RMS of the uncalibrated residual SWD from the model, i.e., the difference in the SWD between the two beams. In general, we find that the RMS error of the SWD is not a critical design parameter, while the ASD requirements, especially at short observation times and low elevation angles, are critical. The focus is therefore on the ASD of the SWD difference between the two beams.

Figure 2 shows the expected ASD of the uncalibrated SWD for two different geometries according to the model. In the following three scenarios of imperfect calibration are presented: beam shape difference, the site position offset, and the pointing offset, respectively.

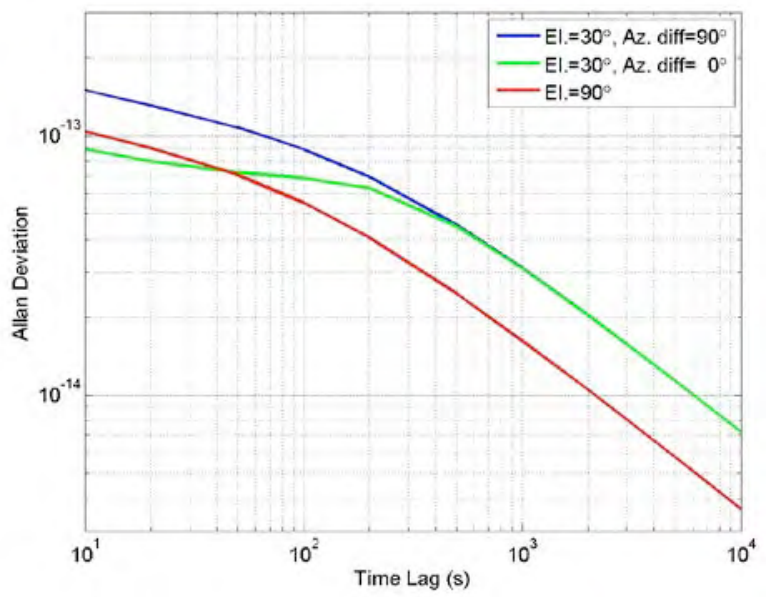

Figure 2: ASD of the uncalibrated slant wet delay at different elevation angles (EI) and wind directions

$(\mathrm{Az})$ with respect to the pointing direction

\subsection{Beam Shape Difference}

An illustration of the DSA and MWR beam shapes are given in Fel! Hittar inte referenskälla.. In order to quantify the effect of the shapes we performed simulations where we modeled the mean wet delay apparent in the two beam volumes, instead of the wet delay apparent in the lines of sight of two ideal "pencil beam" shapes.

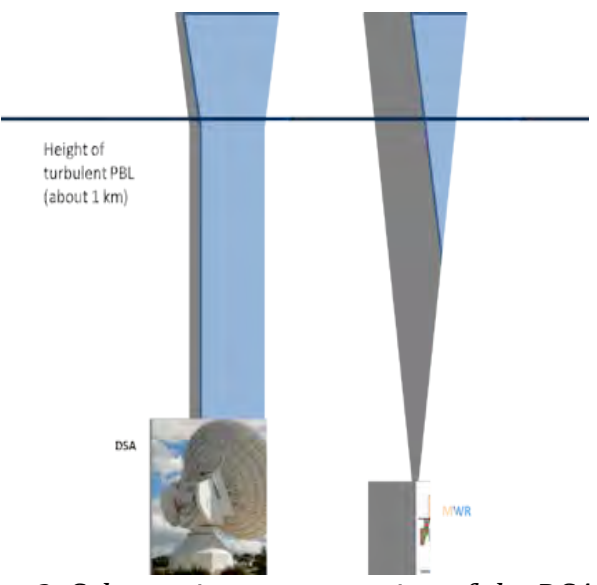

Figure 3: Schematic representation of the DSA and MWR beam shape and dimensions considered for the estimation of the atmosphere variability effects in the Planetary Boundary Layer (PBL)

The MWR beam was modelled as a Gaussian beam with a FWHM (Full Width at Half Maximum) of $1.1^{\circ}$ as resulted from the tradeoff analysis and reported in Section 6.1. On the other hand, the DSA beam was modeled as a cylinder with a diameter equal to $30 \mathrm{~m}$. A couple of different observation geometries were tested for antenna position offsets of 25 and $50 \mathrm{~m}$. We will have a reduction in the ASD of the wet delay due to averaging in the volumes of the beams, although their shapes are significantly different. The reductions in the ASD, when comparing the results for residual SWD in two volumes with those for ideal pencil beams, were fairly similar for all investigated observation geometries. Some results for antenna separation of $25 \mathrm{~m}$ are depicted in Figure 4. The ASD for the observations in the described volumes is of the order of 10-20\% lower than the pencil beam ASD.

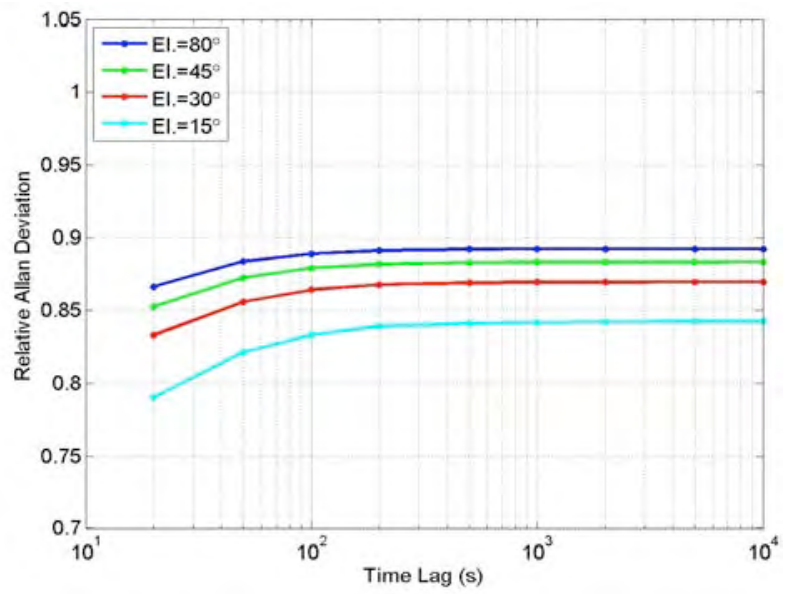

Figure 4: ASD difference presented as ratio between realistic beam shapes and ideal "pencil beam" shapes for the MWR and the DSA antennas separated by $25 \mathrm{~m}$

The similarities in the result for different observation 
geometriesmeans that ASD simulations can be performed assuming pencil beams, and then scaled by, say, 0.86 in order to represent our beam volumes, with relatively little reduction in accuracy.

\subsection{Site Position Offset}

In the simulations, two preliminary design limits were used: the MWR is located at sites that are between 25 and $50 \mathrm{~m}$ away from the DSA. In Figure 5 we present the results at these two position-offset limits when the antennas are pointing in the same direction. The computation of the ASDs for the two distances and for different elevation angles shows that the mission requirements are difficult to meet for a location offset of $50 \mathrm{~m}$, while they are partially satisfied for an offset of $25 \mathrm{~m}$.

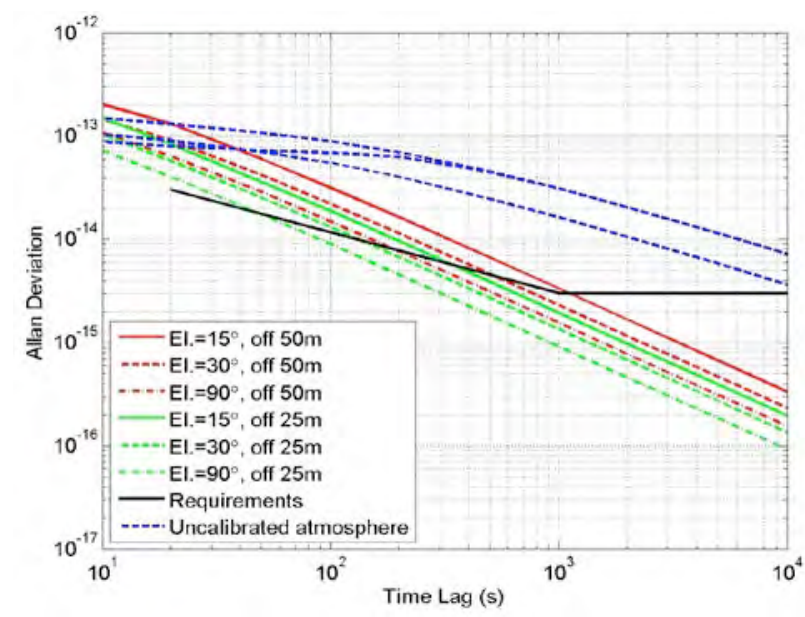

Figure 5: ASD of the residual SWD for site position offsets of 25 and $50 \mathrm{~m}$. The blue curves represents the uncalibrated atmosphere detailed in Figure 2

\subsection{Pointing Offset}

For particular tracking geometries close to the Sun, a pointing offset is sometimes necessary in order to protect the MWR from receiving unacceptable levels of radiations from the Sun.

Different tests have been carried out considering different offset angles. Using the same observation geometry as in the previous analysis, Figure 6 shows the ASD at different elevation angles for the two pointing offsets of $1^{\circ}$ and $2^{\circ}$. The position offset has been set to $0 \mathrm{~m}$ in these simulations. For offset angles larger than $1^{\circ}$ the requirements are difficult to meet. This is further emphasized when observations are carried out at low elevation angles.

It is worth pointing out that under many observation conditions a deliberate pointing offset can actually partly compensate for the (necessary) position offset. When it is possible to let the two beams cross each other in the upper part of the planetary boundary layer the average distance between the points in the two beam volumes become small, resulting in a small residual SWD.

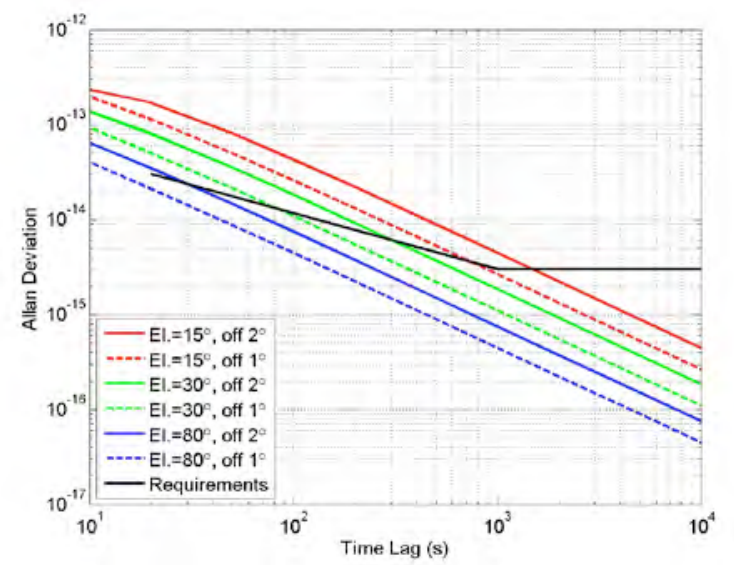

Figure 6: ASD of the residual wet delay for pointing offsets

\section{CHARACTERIZATION OF MWR STABILITY}

The MWRs RPG-ATPROP and RESCOM were installed at Cabauw (NL) site in the framework of the Cabauw Experimental Site for Atmospheric Remote Sensing (CESAR) program [18]. The data acquired have been used for the validation of the characterization methods, described in Sections 2.1, 2.2, and 2.3 to characterize the internal noise of the RPG-ATPROP MWR.

The instruments have been deployed at a distance of about $30 \mathrm{~m}$ apart and pointed in the west direction at an elevation angle of $70^{\circ}$. In a preliminary evaluation of the deploying configuration, it can be assumed that the two instruments are observing the same atmosphere.

Eight months of data were available, but not all of them were suitable for the validation test. Data were selected based on the following criteria: (1) data acquired during clear sky conditions, in order to use the single channel retrieval at the common water vapor channel of 23.8 $\mathrm{GHz}$, (2) continuous intervals of data longer than 6 hours, in order to have a sufficient data for calculation of the ASD at long observation time (10000 s), and (3) concurrent data available from both instruments.

Due to the average meteorological conditions of the site only a few days of data have been used: 18 data sets of different time length.

The presented methods were used to estimate the instrumental stability for $20 \mathrm{~s}$ and $1000 \mathrm{~s}$ observation time. For each procedure the obtained results have been summed up and error bars have been obtained. Figure 7 shows the AWARDS requirements and the 3-sigma error bars. 


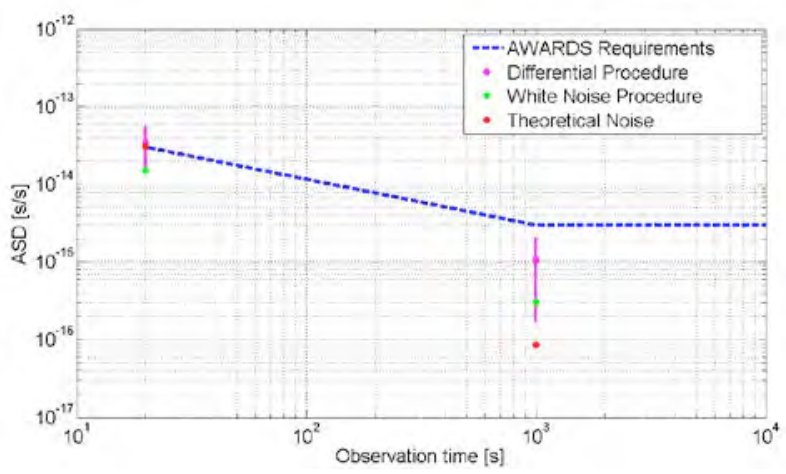

Figure 7: AWARDS requirements (blue) against the results of the differential procedure (pink), the WNP (red) and the theoretical ASD (green)

The data analysis shows that at $20 \mathrm{~s}$ the two error methods result in an overlap. Concerning the theoretical value computed at $20 \mathrm{~s}$, it is in agreement with the other proposed methods and it is also very close to the requirement $\left(3 \times 10^{-14} \mathrm{~s} / \mathrm{s}\right.$ at $\left.20 \mathrm{~s}\right)$. On the other hand at $1000 \mathrm{~s}$ the WNP error bar is overlapped by the error bar of the Differential Procedure, while the theoretical noise is significantly smaller. This is a consequence of the ASD formulation in Eq. (2), and therefore the theoretical noise at $1000 \mathrm{~s}$ is too optimistic.

This result confirms that the proposed methods are in agreement in terms of estimation of the instrumental noise. Moreover, a crucial result is that the RPGATPROP technology is statistically suitable with the AWARDS internal noise requirements. This means that, the current MWR technologies should be considered as the starting point for the design and development of the MORE MCS.

\section{TRADE-OFF ANALYSIS OF THE MORE MEDIA CALIBRATION SYSTEM}

The trade-off analysis carried out during the study is focused on the definition of the main characteristics of the RPG-ATPROP MWR, starting from the results of the stability data analysis. The RPG-ATPROP MWR has been selected as the possible MWR to be installed in the MCS as a state-of-the-art technology. In particular, considering stability results of Figure 7, the internal noise of the RPG-ATPROP MWR at $20 \mathrm{~s}$ observation time, is slightly above the AWARDS requirements. Therefore, the RPG-ATPROP design can be taken as a prototype for the MORE MCS with the exception of the short integration time.

During the analysis, the following effects which could corrupt the performance of the MWR and the entire MCS have been highlighted.

\subsection{Effects at short integration time}

Even if the requirement at $20 \mathrm{~s}$ observation time is almost satisfied, in order to increase the margin in the expected performance, it is proposed to include a double radiometric unit incorporating a total power radiometer and a Dicke-switch radiometer.

In this configuration the atmospheric signal (which is non-polarized in non-precipitating conditions) can be spilt in both orthogonal polarizations. This configuration can be obtained by using an orthomode transducer (OMT) for polarization splitting. A crucial aspect is that OMT is compact and is mechanically much more robust as it is built in waveguide technology and integrated in the protected radiometer housing, Figure 8.

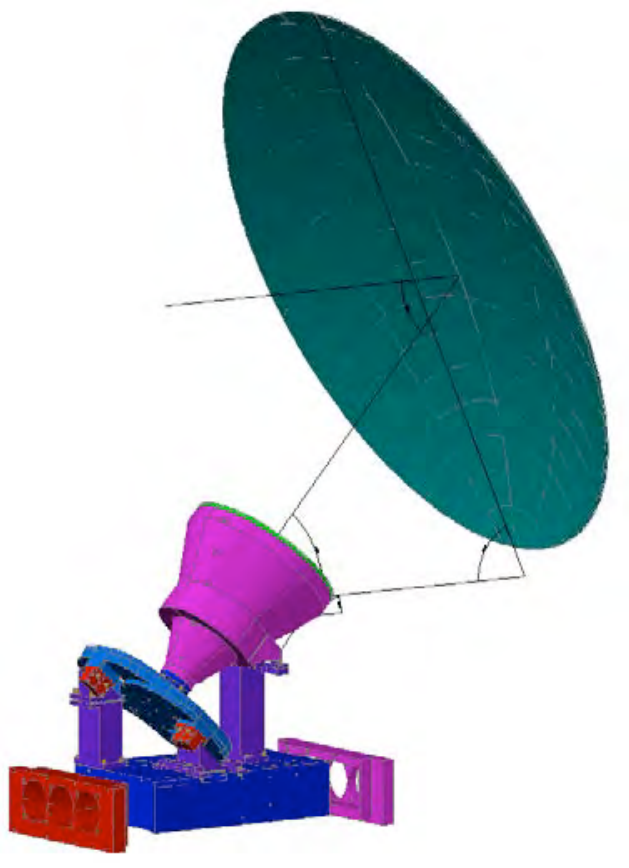

Figure 8: Design of OMT

Because the atmospheric signal is non-polarized in the conditions of interest (no rain) and horn antennas detect one linear polarization only, this approach improves the received signal strength by a factor of two.

In order to be able to calculate the ASD for all relevant time intervals (from $20 \mathrm{~s}$ to $10000 \mathrm{~s}$ ) a weighting procedure for the measurements acquired by both radiometers will have to be developed. However, even if the details of this procedure were not investigated in this study, it was not deemed to represent a major showstopper for the fulfillment of the study goals.

\subsection{Effects of solar contamination}

The effect of the solar contamination represents a criticality for tracking of deep space probes, for both the DSA and the MWR. For this study, this aspect is particularly crucial, due to the orbit of Mercury, the innermost planet in the solar system.

Due to the different beam shape of the DSA and MWR, the presence of the Sun may require a pointing offset, which significantly increases the ASD.

Concerning the DSA, its tracking capability is limited 
when probes are in conjunction with the Sun as demonstrated by the results of the Cassini SCE, even when a multifrequency link was considered. It meant that when the beam was within 6 solar radii from the center of the Sun, the solar plasma effects prevented the ground receivers to lock to the downlink signal. Considering the angular dimension of the Sun of about $0.5^{\circ}\left(R_{\text {Sun }}=0.25^{\circ}\right)$, the minimum angular distance between the beam and the Sun is in the order of $1.5^{\circ}$.

On the other hand, since the MWR will have a wider beamwidth than the DSA, it represents the ultimate limiting factor for experiments taking place during solar conjunctions. For this reason, this aspect drives the design of a new MWR antenna, characterized by smaller beamwidth and suppression of sidelobes (better than $-40 \mathrm{~dB}$ ).

Although the new antenna design will reduce the beamwidth, it is still impossible for the MWR to be pointed as close to the Sun as the DSA. Figure 9 shows a representation of the defined limits around the Sun ( $\delta_{\mathrm{DSA}}$ is the DSA limit and $\delta_{\mathrm{MR}}$ is the MWR limit) and a possible spacecraft path in the sky. The MWR-toSun pointing limit, $\delta \mathrm{MR}$ is defined as the minimum angular distance were the MWR antenna sidelobes are below $-45 \mathrm{db}$.

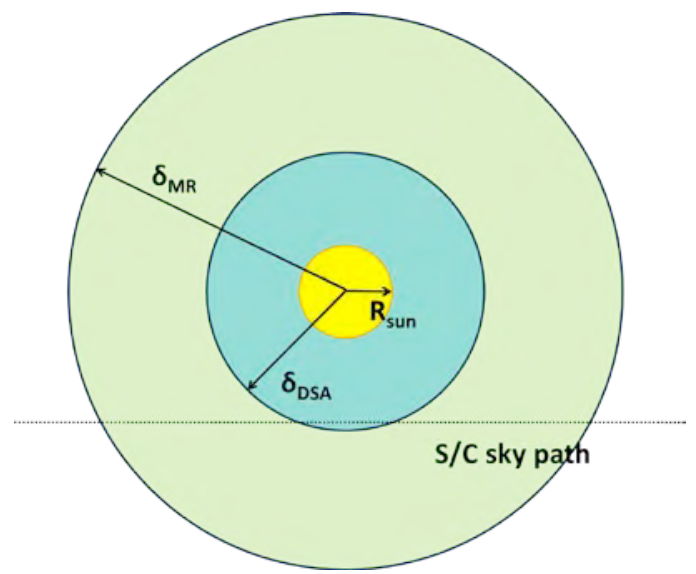

Figure 9: Representation of the pointing boundary limits for both the DSA and MWR

To guarantee tracking and calibration during solar conjunctions, three tracking strategies have been proposed with different pointing offsets. These strategies will be used when the angular distance between the spacecraft and the Sun will be below the MWR limit and are based on the data collected from one or two MWRs.

The proposed strategies are:

-Boundary strategy, the MWR follows the spacecraft position along the MWR boundary circumference as close as possible to the spacecraft position.

-Symmetric strategy, two MWRs have to be considered and they would start to move along their circumference staying aligned together with the position of the probe: one MWR points to the left and the other to the right of the spacecraft position in the sky.

-Equilateral triangle strategy, two MWRs have to be considered, they would move along their boundary circumference in order to create an equilateral triangle where the spacecraft position and the two MWRs are the vertex of the triangle.

In order to appreciate the differences between the strategies Figure 10 shows their comparison in terms of angular distance from the spacecraft to the MWR (yaxis) and spacecraft to the centre of the Sun (x-axis).

It results that the Boundary and Equilateral strategies will reduce the angular distance between the spacecraft and the MWR, while the Symmetric strategy presents a larger distance if compared to the other two.

The Equilateral Triangle strategy would be selected as the nominal strategy to be used if two MWRs are available. On the other hand, the Boundary strategy would be selected as nominal strategy if a single MWR is available or in case of failure of one of the two MWR in the double-MWR configuration.

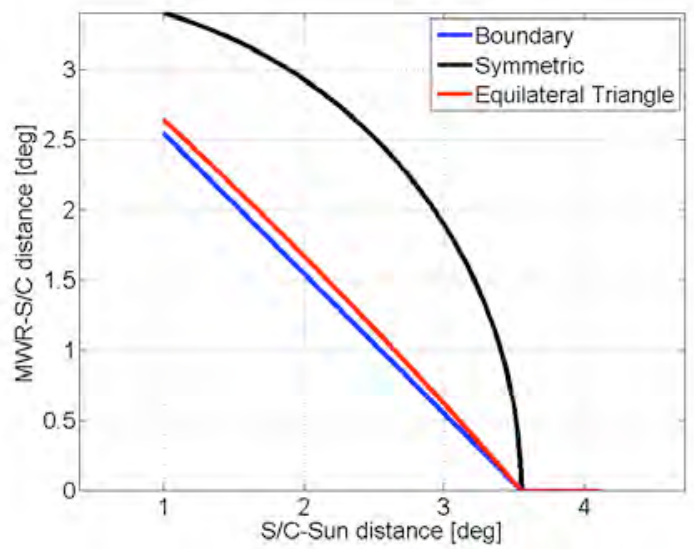

Figure 10: Tracking strategies comparison. Boundary (blue), Symmetric (black) and Equilateral Triangle (red)

Finally, we recommended that the Symmetric strategy would not be implemented in the MWRs due to the introduction of a too large pointing offset, when compared to the Equilateral Triangle strategy.

\subsection{Effects of ground noise emission}

Effect of ground noise emission may be critical for the MWR pointing at low elevation angles. In this study, only elevation angles above $10^{\circ}$ are considered and the emission by the ground can be neglected. This elevation limit is a standard value for the orbit determination computation.

5.4. Effects of the uncertainty of the atmospheric variability

Effects of the atmospheric variability were presented in 
Section 3. Some simulations were carried out considering different tracking geometries for the DSA antenna and an MWR. There is, however, a large uncertainty in the model parameters used in the simulations. For instance, the turbulence parameter $C_{n}^{2}$ can vary significantly with time. Means of quantifying this parameter is essential for the MWR error budget, both in the design phase and later during operation. Several methods exist to estimate $C_{n}^{2}$. A review of methods such as radar, radiosondes, thermosondes, GNSS, and MWR was recently published [19]. A radar system and the launching of sondes are, however, costly activities, which are unrealistic for our application. Instead we propose that existing ground-based GNSS receivers are used to characterize the atmospheric variability during the design phase. Later on, in the operational mode, the use of the MWRs themselves seems to be the optimal choice for this purpose.

Since the wind conditions also will have an impact on the effect of atmospheric variability, the knowledge of them will also be beneficiary for the operation. Therefore, an anemometer can be included in the MCS in order to monitor the wind speed and the wind direction. It should, however, be remembered that the ground conditions often can differ significantly from those higher up in the atmosphere.

\section{ARCHITECTURAL DESIGN OF THE MEDIA CALIBRATION SYSTEM}

The following instruments are proposed to be included in the definition of the MCS: Microwave Radiometers renamed Troposphere Calibration Radiometer (TCR), Atmospheric Profiler, Meteorological Station, Global Navigation Satellite System (GNSS) receiver and Data Acquisition and Processing System.

\subsection{Troposphere calibration radiometer}

The TCR is the most critical component of the MCS and its design is based on the results obtained during the study. The ATPROP MWR has been selected as a baseline to define a preliminary design for possible development, however, there are two notable changes: (1) the need for a smaller antenna beamwidth with about $1^{\circ}$ Full Width Half Maximum (FWHM) with a high side lobe suppression to achieve the requirements for atmospheric beam matching and to avoid excess radiation from the Sun, (2) the inclusion of a total power receiver in addition to the Dicke switch noise injection receiver in order to achieve the ASD requirements for both short and long integration times. Figure 11 illustrates the planned setup of the core TCR. In the following the different functional blocks will be described.

The TCR will include the seven K-band frequencies of ATPROP to characterize the SWD and water vapour variations. In ATPROP the atmospheric signal is divided in both orthogonal polarizations with one going to the K-band receiver and the other one to another receiver (in the $50-60 \mathrm{GHz}$ frequency band) for temperature profiling.

In the MORE TCR, the V-band receiver is replaced by a second $\mathrm{K}$-band receiver that is identical to the first but does not use Dicke switching. In this way, redundancy is achieved. In case of failure of one receiver the other will still provide useful information.

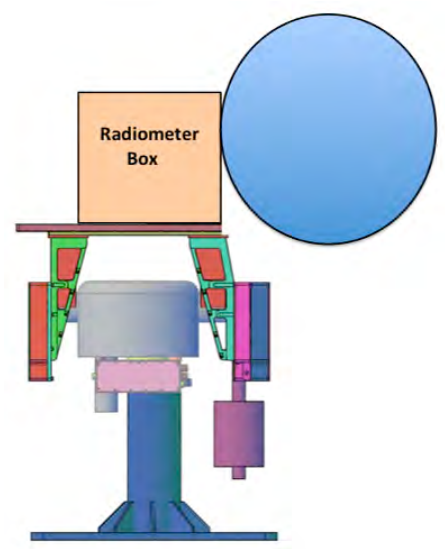

Figure 11: Possible setup of the TCR with an $80 \mathrm{~cm}$ antenna and radiometer units, i.e. the K-band module (total-power and the Dicke system) and an (optional) temperature profiler

The radiometer box contains the microwave receiver units at K-band and an OMT is directly mounted at the exit of the corrugated horn antenna. The waveguide for vertical polarization (V-channel) transfers the signal to a microwave receiver unit which is identical to ATPROP. Here the signal is calibrated using a Dicke switch and a noise diode injection. The horizontal polarization $(\mathrm{H}-$ channel) is transferred into a second receiver unit which is identical except the omission of the Dicke switch.

The receiver units contain the same low noise amplifier (LNA) and filterbank system used in ATPROP. Each unit has seven channels that are individually amplified and bandpass filtered. Note that the filters have to be sharp in bandwith to reduce the risk to get Radio Frequency Interference (RFI) contamination. The receiver noise temperature of the channels will be lower than $400 \mathrm{~K}$.

Each frequency channel has its own detector. Their outputs are coupled via a Multiplexer (MUX) to a 16 bit Analog to Digital Converter (ADC). A digital Input/Output (I/O) interface combines both microwave receiver units ( $\mathrm{V}$ and $\mathrm{H}$ channel) and the internal computer. This computer then stores raw data, controls calibration and positioning and communicates with the external Data Acquisition and Processing System.

\subsection{Atmospheric profiler}

In order to define an accurate retrieve procedure to estimate the SWD at the DSA a dedicated instrument 
capable to estimate the air temperature and humidity vertical profiles is required.

The installation of a dedicated profiler would allow an autonomously scan pattern, without limitation to the TCR's tracking activity. In general, while the TCR will be synchronized to track in parallel with the DSA, the profiler can continuously perform boundary layer scan and provide information on atmospheric profiles for the assessment of the retrieval algorithm. Moreover, it can be pointed in any direction for the most accurate definition of the atmospheric profiles. As an example, an instrument well suited for this aspect is the RPGHATPRO, equipped with 6 channels in the water vapor one in the liquid and 7 in the oxygen line [20].

\subsection{Meteorological station}

Another important component of the entire MCS is represented by the meteorological station. In general, it shall include a surface temperature sensor, a surface humidity sensor, a surface pressure sensor, a wind anemometer, a ceilometer and a rain sensor.

Concerning the temperature, humidity, pressure and rain rate parameters, their measurements are crucial for the setup of the TCR retrieval algorithm in order to accurately estimate the slant path delay. On the other hand, the accurate estimation of the surface pressure is enough to estimate the hydrostatic path delay.

The wind sensor is important to estimate the strength of turbulence and to evaluate it. At present, Cebreros complex, as well as all the other ESA/ESTRACK complexes, are equipped with a meteorological station which is currently used to provide the standard troposphere path delay data for deep ESA's deep space probe tracking activity.

\subsection{Global Navigation Satellite System}

In the MCS an optional component is a GNSS receiver. It can serve as a backup instrument for different applications: estimation of atmosphere variability, time reference and monitoring of Zenith Total Delay (ZTD) [21]. Provided that accurate observations of the ground pressure is acquired, the equivalent Zenith Wet Delay (ZWD) can be inferred as a reference in order to validate the performance of the microwave radiometers. A geodetic dual-frequency receiver is required in order to reduce and suppress error sources of the GNSS. The dual frequencies are for example needed to estimate and remove the dispersive effect on the propagation delay of the signal caused by the free electrons in the ionosphere. This kind of receivers are usually installed on concrete monuments in order to provide high position stability and equipped with a choke ring antenna, to suppress the multipath effects from the ground as well as from other nearby objects.

\subsection{Data acquisition and processing system}

Another important component is represented by the data acquisition and processing system. This system is crucial to collect data from all the instruments of the MCS and to process them in order to obtain a more accurate calibration for deep space probe tracking purposes. This system provides the connection of the MCS data directly to the ESA ESOC Flights Dynamics team.

Some MCS instruments, as the TCRs, are able to operate independently using its embedded computer. Once the TCRs have been set up they are capable to perform the tracking activity synchronized with the DSA. Then, data are sent to an external computer which interfaces with all the other MCS instruments and processes all the data in order to provide the troposphere calibration data.

The data acquisition and processing system software needs to control the activity of all the instruments. Concerning the TCRs the software has to manage automatic tipping curve procedure such that no interference with times important for spacecraft tracking are used. At the same time the software has to manage the scanning activity of the profiler as well as to monitor the acquisition activity of the other instruments.

\subsection{Deployment Configurations}

Following the list of equipment described in the previous sections, two possible configurations of the MCS have been foreseen, characterized by the installation of one or two twin TCRs.

Among all possible configurations, some aspects have to be fixed for a correct deployment of the TCS, in order to avoid blockage of the field of view of the MWRs due to the DSA dish.

The DSA tracks deep space spacecraft along the ecliptic plane. In particular, this means that the TCR would be southward if the DSA site is in the northern hemisphere or northward if the DSA site is in the southern hemisphere.

Generally speaking, installing a MWR co-located in the DSA subreflector would provide the most accurate estimation of the real path delay observed by the DSA. Nevertheless, several technological and operational issues have to be considered with this option. Therefore, the preferred choice at present is to have the TCR installed on the ground as close as possible to the DSA. In order to mitigate the effects of the atmospheric fluctuations, the previous analysis has shown that the deployment distance between the TCRs and the DSA basement should be limited to maximum $25 \mathrm{~m}$ (see Section 4). Figure 12 shows a possible deployment of the MCS configuration at Cebreros, where the two MWRs are southward of the DSA antenna dish at a distance of about $25 \mathrm{~m}$ from the center of the DSA basement.

The distance between the two TCRs has been fixed to 
$20 \mathrm{~m}$. This distance is crucial to guarantee a redundancy without loss of tracking data in case of failure. In particular, the distance between the two TCRs could be reduced without limitations (with the exception of the potential screening effect of one TCR with respect to the other) but could not be increased indefinitely, because of possible blockages of the field of view of the instruments at low elevation angles.

In order to better characterize the state of atmosphere, an additional radiometer profiler has been deployed between the two TCRs.

To complete the MCS, a GNSS receiver and a meteorological station have to be installed at the ground station complex.

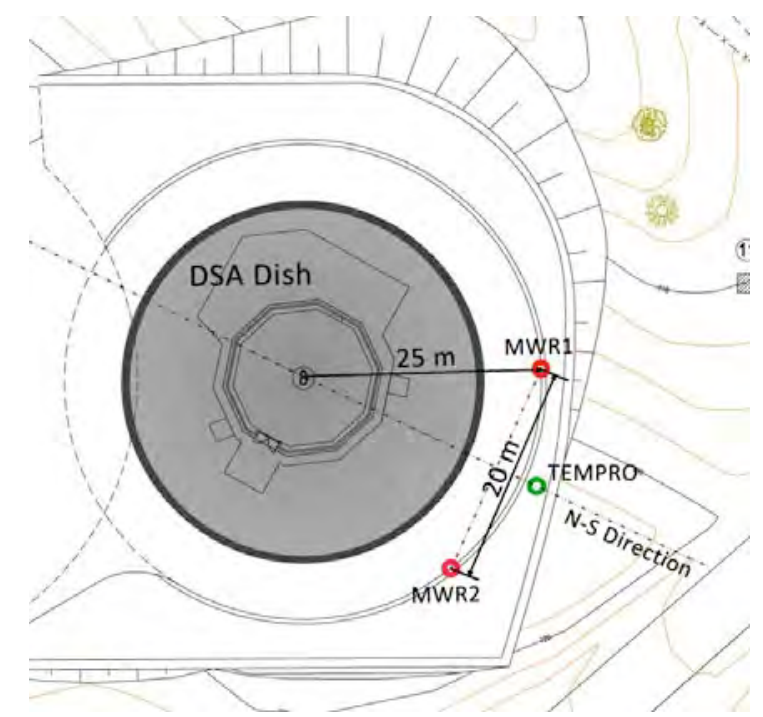

Figure 12: Overview of a possible deployment of the MCS with two MWR in Cebreros

\section{CONCLUSIONS AND FUTURE WORK}

The study demonstrates the need of an accurate and stable MCS, based on MWR technology, in order to satisfy the stringent radio link stability requirements for MORE. The RPG-ATPROP MWR was used as a reference state-of-the-art MWR and it has been characterized in terms of internal stability and overall ASD. In particular, the capability of the RPG-ATPROP to satisfy the MORE requirements at $1000 \mathrm{~s}$ and $10000 \mathrm{~s}$ observation time was demonstrated. On the other hand, at $20 \mathrm{~s}$ observation time the requirement is not fully met.

The data analysis led to the definition of a new MWR named Troposphere Calibration Radiometer, to be the main component of the MORE MCS. The TCR will be based on a combination of Dicke switch and total power radiometer units and equipped with an $80 \mathrm{~cm}$ diameter reflector antenna.

The analysis led also to the definition of two MCS configurations: based on the installation of one or two TCRs. The suggested configuration includes two TCRs and offers a better capability to mitigate Sun contamination effects, ground emission and atmospheric variability.

Among the good results obtained during the study, further aspects have been highlighted and additional investigations are required. In particular the small amount of meteorological data in Cebreros did not allow the possibility to correctly determine representative statistics for the $C_{n}^{2}$ turbulence parameter.

The study has provided guidelines for the system design. However certain parameters (e.g. the distance between the radiometer(s) and the DSA, the relative distance between the two radiometers - if such an option is selected) are not to be considered final.

In addition, two issues need further investigations, namely: the way to combine the information of the total power MWR (for short integration times, up to approx $100 \mathrm{~s})$ and the Dicke switch MWR for longer integration times into a single "synthetic" observable, and an accurate simulation for the evaluation of the benefits of a crossing beam configuration in order to mitigate the atmospheric variability effect due to a position offset between the DSA and the MWR.

\section{ACKNOWLEDGMENT}

The AWARDS team wants to thank the experts working at Cebreros ESA DSA-2 site for their support during the study.

\section{REFERENCES}

1. Tortora P., et al, "AWARDS - Advanced microwave Radiometers in Deep space Stations", Final Report, ESTEC Contract No. 23127/10/NL/LvH, v1.0, 15/12/11 2. Asmar, S.W., "Radio as a Science Tool", Proceedings of the IEEE, Vol. 98, Issue 10, 2010, doi:10.1109/JPROC.2010.2060247, pp. 1686-1687.

3. Asmar, S.W., Armstrong, J.W., Iess, L., Tortora, P., "Spacecraft Doppler Tracking: Noise Budget and Accuracy Achievable in Precision Radio Science Observations" Radio Sci., Vol. 40, Issue 2, 2005, doi:10.1029/2004RS003101, pp. 1-9.

4. Armstrong, J. W., Iess, L., Tortora, P., Bertotti, B., "Stochastic Gravitational Wave Background: Upper Limits in the $10^{-6}$ to $10^{-3} \mathrm{~Hz}$ Band", Astrophys. J., Vol. 599, 2003, pp. 806-813.

5. Bertotti B., L. Iess and P. Tortora, "A test of general relativity using radio links with the Cassini spacecraft", Nature, vol. 425, 2003, pp. 374-376.

6. Iess, L., Asmar, S., Tortora,P. "MORE: An advanced tracking experiment for the exploration of Mercury with the mission BepiColombo", Acta Astronautica, Vol. 65, Issue 5-6, 2009, pp. 666-675, DOI:10.1016/j.actaastro.2009.01.049

7. Resch G.M, Bar-Sever Y, Keihm S.J, Kroger P, Linfield R.P, Mahoney M.J, Tanner A, Teitelbaum L.P, "Atmospheric calibration for precision Doppler 
9. Elgered G., "Tropospheric radio path delay from ground-based microwave radiometry" Atmospheric Remote Sensing by Microwave Radiometry (M. Janssen, editor), New York: Wiley \& Sons, 1993

10. Nörenberg D., S. Crewell, U. Löhnert, Th. Rose, A. Martellucci "A novel ground-based microwave radiometer for high precision atmospheric observations between 10 and $90 \mathrm{GHz}$ ", IEEE IGARSS, Boston, MA, USA, 6-11 July, 2008, DOI: 10.1109/IGARSS.2008.4779500

11. Dicke R.H., "The Measurement of Thermal Radiation at Microwave Frequencies" Rev. Sci. Instr. Vol. 17, pp. 268-275, 1946

12. Thornton C. L. and J. S. Border, Radiometric Tracking Techniques for Deep-Space Navigation, Deep Space Communications and Navigation Series, Monograph 1, Jet Propulsion Laboratory, October 2000 13. Löhnert U. and S. Crewell, "Accuracy of cloud liquid water path from ground-based microwave radiometry. Part I. Dependency on Cloud model statistics“. Radio Sci., Vol. 38 Issue 3, 8041, 2003, doi:10.1029/2002RS002654.

14. Keihm S. J., A. Tanner and H. Rosenberger, "Measurements and Calibration of Tropospheric Delay at Goldstone from the Cassini Media Calibration System”, IPN Progress Report 42-158, August 15, 2004 15. Tanner A. B., "Development of a high stability water vapor radiometer", Radio Sci., Vol. 33, Issue 2, pp. 449-462, 1998

16. Camps, A. and J. M. Tarongi, "Microwave radiometer resolution optimization using variable observation time," Remote Sensing, Vol. 2, No. 7, 1826-1843, 2010. doi:10.3390/rs2071826

17. Treuhaft, R. N., and G. E. Lanyi, "The effect of the dynamic wet troposphere on radio interferometric measurements", Radio Sci., 22 (2), 251-265, doi:10.1029/RS022i002p00251, 1987.

18. Russchenberg $\mathrm{H}$, et al. "CESAR: Cabauw Experimental Site for Atmospheric Remote Sensing", Proceedings of the XXVIIth General Assembly of the International Union of Radio Science, 17-24 Aug. 2002 19. Nilsson, T., and R. Haas, "Impact of atmospheric turbulence on geodetic very long baseline interferometry", J. Geophys. Res., 115, B03407, 2010, doi:10.1029/2009JB006579.

20. Rose, T, et al., "RPG-HATPRO and Profiling Radiometers", RPG Publications, http:// www.radiometer-physics.de

21. Bevis M., S. Businger, T. A. Herring, C. Rocken, R. A. Anthes and R. H. Ware, "GPS meteorology: remote sensing of atmospheric water vapor using the Global Positioning System", J. Geophys. Res., Vol. 97, pp. 